\title{
Cerebrospinal fluid biomarkers in various pediatric neurologic diseases
}

Jeongho Lee, MD, PhD

Department of Pediatrics, Soonchunhyang University Seoul Hospital, Seoul, Korea

\section{Key message}

Cerebrospinal fluid (CSF) has many important biomarkers that are commonly analyzed in pediatric neurologic diseases, including central nervous system infection and inflammation. Neurologic disease in pediatrics is difficult to diagnosis, there are challenges in developing CSF profiles. Some biomarkers are expected to help differential diagnosis.

The analysis of cerebrospinal fluid (CSF) is an important but invasive diagnostic test performed in young children. Since the introduction of the lumbar puncture test by Dr. Heinrich Quincke in the 19th century, CSF biomarkers have been used in pediatric neurology. ${ }^{1)}$ The CSF analysis biomarkers frequently used by pediatric neurologists include cell count, albumin ratio with CSF/blood, lactate, immunoglobulin G (IgG), and immunoglobulin M.2)

CSF components have been evaluated as biomarkers of adult neurological disorders, including Alzheimer's disease, traumatic brain injury (TBI), amyotrophic lateral sclerosis, and normalpressure hydrocephalus. ${ }^{1-3)}$ Because lumbar puncture is an invasive test, it is difficult to analyze CSF profiles in healthy young children and identify CSF biomarkers in pediatric neurological disorders. MicroRNAs (miRNAs) in the CSF are promising biomarkers for neurological diseases. ${ }^{4,5)}$ Although few CSF studies have examined miRNAs for pediatric neurologic disease, it is meaningful to focus on them in pediatric patients with various neurological disorders. Low-absorbing polypropylene tubes are recommended to be used to collect CSF samples and can be left at room temperature for as long as 30 minutes. Transport time to the laboratory must be minimized and the sample be cooled on the ice during transport. ${ }^{2)}$

Basic CSF profiles, including cell count, protein level, CSF/ plasma albumin ratio, lactate level, and culture are used to diagnose patients with central nervous system (CNS) infections and inflammation. Other candidate biomarkers are neopterin, tau, glial fibrillary acidic protein (GFAP), and neurofilament light (NFL) in patients with infectious and inflammatory CNS

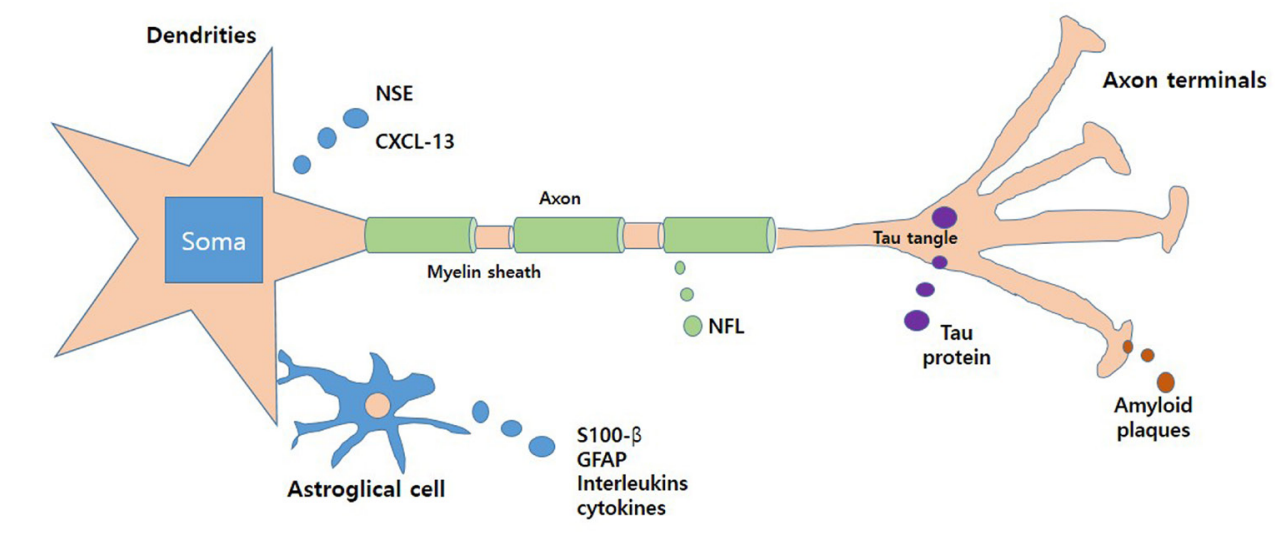

Inflammation cells cytokines, IgG, IgM

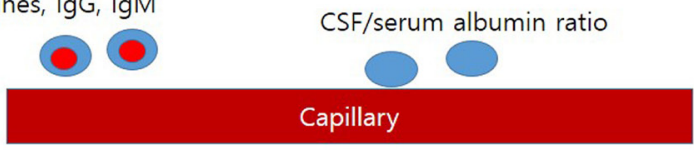

Fig. 1. Schematic illustration of cells in the brain and CSF biomarkers. NSE, neuron-specific enolase; CSF, cerebrospinal fluid; CXCL-13, chemokine ligand 13; GFAP, glial fibrillary acidic protein; Ig, immunoglobulin; NFL, neurofilament light; S-100ß, S-100 calcium-binding protein beta. 
conditions. ${ }^{2,6)}$ Microglia and astrocytes form neopterin after cellrelated interferon-gamma. White matter and myelinated axons have high structural proteins such as NFL, a good indicator of CNS infection and injury.,7) MiR-29a is a candidate biomarker for CNS infection, especially tuberculous meningitis. Several studies have reported acute disseminated encephalomyelitis (ADEM) and multiple sclerosis (MS) in the first clinical episode of neurological symptoms. CSF leukocyte count, IgG level, and oligoclonal bands are useful but nonspecific biomarkers of CNS inflammation. Chemokine ligand 13 is a new candidate biomarker in children with ADEM and MS versus those without inflammation. ${ }^{2)}$ Few studies have shown the elevation of miRNA profiles (miR-4486, miR6850-5p, miR-642b-3p, miR-7107-5p, and miR4281) in patients with febrile seizure. ${ }^{2,8)}$

In the neonatal period, hypoxic-ischemic encephalopathy (HIE) is the most common cause of perinatal mortality and longterm disability. Neuron-specific enolase (NSE), interlukin-6, neurofilament protein, and S-100 are related with HIE. A few studies showed that CSF NSE level predicted brain damage severity in hypothermia-treated HIE. ${ }^{\text {9) }}$

Famous biomarkers associated with axonal changes such as TBI are tau and S-100 calcium-binding protein beta (S-100/). These were found in children with seizures and encephalopathy. GFAP and S-100 $\beta$ are also expressed by astrocytes, after TBI, and in cases of neuroinflammation. In pediatric patients with TBI, the levels of platelet selectin (P-selectin) are elevated due to ischemic and reperfusion events. Vascular endothelial growth factor is a neuroprotective molecule in experimental brain injury models whose levels were increased after TBI in human and animal studies. ${ }^{10)}$ Other various biomarkers were found in CNS tumors, intraventricular hemorrhage, and congenital hydrocephalus. A number of tumor-related biomarkers including circulating tumor DNA (ctDNA), miRNAs, insulin-like growth factor (IGF), IGFbinding protein, prostaglandin D2, and apolipoprotein A-II. ${ }^{2)}$ (Fig. 1)

Neurotransmitters are important biomarkers for many neurological diseases related to inborn errors of metabolism. Serotonin, dopamine, and tetrahydrobiopterin (BH4) metabolites are key markers for the diagnosis of $\mathrm{BH} 4$ deficiency and other metabolic diseases.

Several CSF markers may be used to diagnose and monitor treatments. After identifying proper biomarkers and laboratory standardization, it is widely used in pediatric neurology.
See the article "Promising candidate cerebrospinal fluid biomarkers of seizure disorder, infection, inflammation, tumor, and traumatic brain injury in pediatric patients" via https://doi. org/10.3345/cep.2021.00241.

\section{Footnotes}

Conflicts of interest: No potential conflicts of interest relevant to this article are reported.

\section{References}

1. Limbrick DD Jr, Castaneyra-Ruiz L, Han RH, Berger D, McAllister JP, Morales DM. Cerebrospinal fluid biomarkers of pediatric hydrocephalus. Pediatr Neurosurg 2017;52:426-35.

2. Kim SH, Chae SA. Promising candidate cerebrospinal fluid biomarkers of seizure disorder, infection, inflammation, tumor, and traumatic brain injury in pediatric patients. Clin Exp Pediatr 2022;65:56-64.

3. Shahim P, Månsson JE, Darin N, Zetterberg H, Mattsson N. Cerebrospinal fluid biomarkers in neurological diseases in children. Eur J Paediatr Neurol 2013;17:7-13.

4. Mitchell PS, Parkin RK, Kroh EM, Fritz BR, Wyman SK, PogosovaAgadjanyan EL, et al. Circulating microRNAs as stable blood-based markers for cancer detection. Proc Natl Acad Sci U S A 2008;105:105138.

5. Sheinerman KS, Umansky SR. Circulating cell-free microRNA as biomarkers for screening, diagnosis and monitoring of neurodegenerative diseases and other neurologic pathologies. Front Cell Neurosci 2013;7: 150.

6. Casas-Alba D, Valero-Rello A, Muchart J, Armangué T, Jordan I, Cabrerizo M, et al. Cerebrospinal fluid neopterin in children with enterovirus-related brainstem encephalitis. Pediatr Neurol 2019;96:70-3.

7. Kristiánsdóttir R, Uvebrant P, Rosengren L. Glial fibrillary acidic protein and neurofilament in children with cerebral white matter abnormalities. Neuropediatrics 2001;32:307-12.

8. Kim SH, Yun SW, Kim HR, Chae SA. Exosomal microRNA expression profiles of cerebrospinal fluid in febrile seizure patients. Seizure 2020; 81:47-52.

9. León-Lozano MZ, Arnaez J, Valls A, Arca G, Agut T, Alarcón A, et al. Cerebrospinal fluid levels of neuron-specific enolase predict the severity of brain damage in newborns with neonatal hypoxic-ischemic encephalopathy treated with hypothermia. PLoS One 2020;15:e234082.

10. Shore PM, Jackson EK, Wisniewski SR, Clark RS, Adelson PD, Kochanek PM. Vascular endothelial growth factor is increased in cerebrospinal fluid after traumatic brain injury in infants and children. Neurosurgery 2004;54:605-11; discussion 11-2.

How to cite this article: Lee J. Cerebrospinal fluid biomarkers in various pediatric neurologic diseases. Clin Exp Pediatr 2022;65:81-2. https://doi.org/10.3345/cep.2021.01445 